\title{
Estudo das propriedades de misturas de excipientes farmacêuticos
}

\author{
M. U. ROCHA \& R. CONDOTTA \\ Centro Universitário da FEI, Departamento de Engenharia Química. \\ E-mail para contato: rcondotta@fei.edu.br
}

\begin{abstract}
RESUMO - Na maioria dos processos industriais é encontrada uma grande diversidade de materiais pulverulentos. $\mathrm{O}$ conhecimento das propriedades associadas à escoabilidade destes materiais é de extrema importância, pois podem sofrer alterações em suas características durante o processamento, estocagem e transporte, sendo a avaliação deste parâmetro de grande importância nas operações industriais e nos projetos de equipamentos de processamento de pós. A microcelulose e lactose são exemplos de materiais pulverulentos amplamente utilizados na indústria farmacêutica para a produção de comprimidos e capsulas. Suas aplicações são semelhantes, entretanto sua natureza e, consequentemente, suas propriedades físico-químicas são distintas. Neste trabalho estudou-se de algumas propriedades físicas inerentes ao escoamento de microceluloses 101 e 102 e de lactoses monohidratada e "spray-dried", puras e em misturas contendo diferentes teores de materiais de cada natureza. Foram realizados testes em diferentes condições de consolidação, de maneira a representar as reais condições das diversas etapas do processo produtivo.
\end{abstract}

\section{INTRODUÇÃO}

Muitas substâncias possuem estabilidade quando estão em formato sólido, apresentando grande facilidade no manuseio, transporte e armazenamento. Apesar dos materiais particulados apresentarem um escoamento que se assemelha ao dos líquidos, a taxa de escoamento não é constante, pois podem sofrer alterações em suas características durante o processamento.

Um pó ou material pulverulento pode ser definido como um conjunto de partículas pequenas de origem, estrutura e composições variadas, que definem suas propriedades físicoquímicas. A falta de conhecimento neste tema pode resultar em perda de produção, riscos para a saúde, má qualidade do produto, explosão ou colapso em seu armazenamento.

Geralmente, a análise da fluidez de insumos farmacêuticos é realizada através de ensaios experimentais em estado estáticos ou dinâmicos sob baixas tensões de consolidação, o que não representa a condição exata dos insumos no processo. Ao ser submetido à tensões elevadas, a coesão desenvolvida em um material pulverulento torna-se uma importante propriedade a ser investigada, entretanto poucos são os métodos que permite sua medida com exatidão.

Com o intuito de estudar os principais elementos que afetam a fluidez e interferem nas propriedades físicas essenciais, como densidade e porosidade, e outras propriedades 
fenomenológicas (dinâmicas), como fluxo variável, aeração e cisalhamento, dos materiais pulverulentos, neste trabalho inferiu-se o comportamento de algumas substancias comumente empregado no segmento farmacêutico, como lactose e microcelulose.

\section{REVISÃO BIBLIOGRÁFICA}

Os excipientes farmacêuticos são substância auxiliares incorporada aos medicamentos que acompanham o princípio ativo para dá-lhe formula, massa ou volume, tornando-o adequado ao consumo. Podem ser do tipo: lubrificantes, corantes, aglutinantes, aromatizantes, entre outros. Dessa forma, para impedir problemas, como degradação do fármaco, presença de impurezas, testes para avaliar a funcionalidade dos excipientes devem ser feitos, com a intenção de analisar suas propriedades física, físico-químicas e biofarmacêuticas (Ferreira, 2013).

Já os fármacos, conhecidos como IFAs ou drogas, são as substancias químicas ativas empregadas na fabricação de um medicamento, sendo responsáveis pela ação farmacológica (FARMACOPÉIA BRASILEIRA, 2010).

Dentro os fármacos, os comprimidos são os mais comuns no mercado, pois possuem maior estabilidade e facilidade no transporte. No processamento de comprimidos deve-se levar em conta o escoamento dos pós. Uma fluidez adequada é essencial para que haja um preenchimento rápido e eficiente das matrizes em compressoras rotativas (Prista, 2002).

Existem diversos fatores que podem exercer influência na fluidez de pós, destacandose: umidade, morfologia das partículas, propriedades de superfície, tamanho e distribuição de tamanho das partículas, densidade, dentre outros (Kibbe, 2009).

Dentre os excipientes mais comuns no processo de fabricação de comprimidos, destacam-se a lactose a microcelulose.

Lactose: É fabricada por cristalização e secagem, seguida de um processamento secundário para se criar uma variedade de produtos disponível para escolha.

Microcelulose: É produzida por hidrólise parcial de polpa de madeira de alta pureza. A alta compactabilidade da MCC torna-a uma das mais usadas em formulações de fármacos, porém apresenta baixa escoabilidade.

\section{METODOLOGIA}

\subsection{Materiais}

Os insumos farmacêuticos utilizados neste trabalham forma as Microcelulose 101 e 102, a Lactose Monohidratada e a Lactose seca em Spray-Drier, cedidas pela empresa Farmanguinhos/RJ. Misturas das duas famílias de produtos foram confeccionadas, conforme apresentado na Tabela 1.

Tabela 1: Misturas de excipientes utilizadas e suas respectivas composições. 


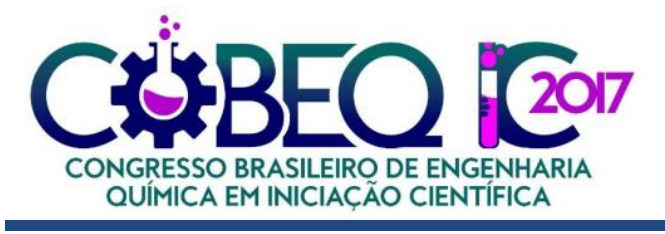
XII Congresso Brasileiro de Engenharia Química
em Iniciação Científica
UFSCar - São Carlos - SP
16 a 19 de Julho de 2017

\begin{tabular}{|c|c|c|c|}
\hline \multirow{2}{*}{ Mistura } & Lactose & \multicolumn{2}{|c|}{ Microceluose } \\
\cline { 3 - 4 } & Monohidrat. & 101 & 102 \\
\hline $\mathbf{1}$ & $60 \%$ & $40 \%$ & \\
\hline $\mathbf{2}$ & $40 \%$ & $60 \%$ & \\
\hline $\mathbf{3}$ & $60 \%$ & & $40 \%$ \\
\hline $\mathbf{4}$ & $40 \%$ & & $60 \%$ \\
\hline
\end{tabular}

\begin{tabular}{|c|c|c|c|}
\hline \multirow{2}{*}{ Mistura } & \multirow{2}{*}{$\begin{array}{c}\text { Lactose } \\
\text { Spray-dried }\end{array}$} & \multicolumn{2}{|c|}{ Microcelulose } \\
\cline { 3 - 4 } & $60 \%$ & 101 & 102 \\
\hline $\mathbf{5}$ & $60 \%$ & $60 \%$ & \\
\hline $\mathbf{6}$ & $40 \%$ & \\
\hline $\mathbf{7}$ & $60 \%$ & & $40 \%$ \\
\hline $\mathbf{8}$ & $40 \%$ & & $60 \%$ \\
\hline
\end{tabular}

\subsection{Caracterização das Amostras}

Inicialmente as amostras sofram submedidas à análise granulométrica com auxílio do analisador de partícula Bluewave (MicroTrac) em via úmida, usando etanol anidro como meio dispersante. Já a morfologia das partículas foi analisada com auxílio do microscópio eletrônico de varredura CamScan CS 3200 LV (Cambridge).

Em seguida foram determinadas as densidades das amostras, para posterior estimativa da porosidade da mesma. As densidades reais das partículas foram obtidas com auxílio do aparelho Gas Pycnometer, modelo AccuPyc II 1340, da marca Micromeretics, empregando gás hélio.

As densidades aparente e compactada foram obtidas com auxílio do aparelho AutoTap, modelo TAP-2S, da marca Logan, segundo a metodologia USP-II. Nesta análise, foram introduzidos cerca de $80 \mathrm{ml}$ de amostra numa proveta graduada de $100 \mathrm{ml}$, fixada à base do aparelho. A densidade aparente foi obtida a partir desta condição inicial e, em seguida, programou-se o aparelho para realizar até 2500 batidas, que permitiu a obtenção da densidade compactada. Os testes foram realizados em duplicatas. A relação das medias destas densidades foi utilizada para determinação do índice de Hausner (FARMACOPÉIA BRASILEIRA, 2010)

\subsection{Reômetro de Pó FT-4}

O aparelho FT-4 (Freeman Technology) é um reômetro de pó capaz de medir a força axial e rotacional (torque), através de uma lamina torcida de $48 \mathrm{~mm}$ de comprimento quando essa se movimenta pelo leito, como ilustrado na Figura 1.

Figura 1: (a) força axial e rotacional (torque); (b) rotação nos sentidos anti-horário e horário, respectivamente; (c) experimentos de aeração com injeção de ar na base do recipiente.

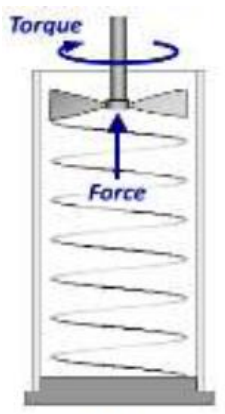

(a)

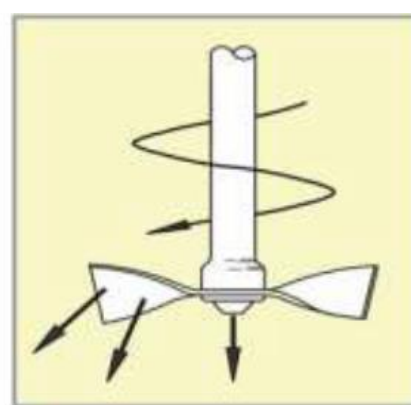

(b)

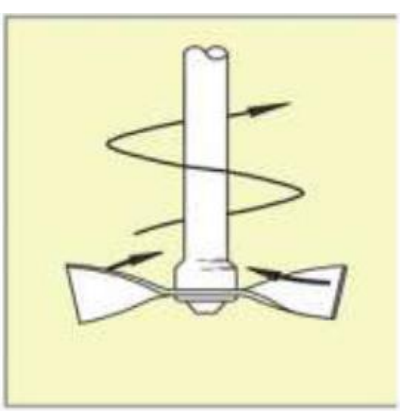

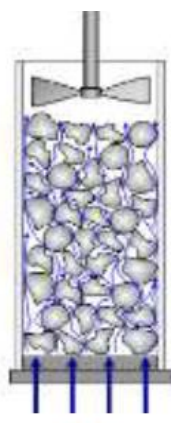

(c)

Teste de estabilidade, energia básica (BFE) e fluxo variável (VRF): Neste teste, $160 \mathrm{ml}$ de amostra contida num cilindro de $50 \mathrm{~mm}$ de diâmetro é permeada pela lâmina a uma 
velocidade constante da ponta da lâmina de $100 \mathrm{~mm} / \mathrm{s}$, por 7 vezes. Ao fim das 7 medidas, obtém-se o valor da energia básica de fluxo (BFE) e inicia-se o teste do fluxo variável (VRF) com quatro pontos, onde a velocidade da ponta da lâmina é modificada para 100, 70, 40 e 10 $\mathrm{mm} / \mathrm{s}$, verificando a variação de energia necessária para movimentar a amostra.

Teste de aeração: é realizado de maneira análoga ao teste de fluxo variável, mas com ar sendo injetado na base do recipiente, com a finalidade de se comparar as propriedades do pó na forma aerada e não aerada.

Teste de cisalhamento: Foi realizado 2 testes de cisalhamento para todas as amostras, na tensão de consolidação de 3,6,9 15kPa (em duplicatas) para a determinação do plano de ruptura, no intuito de se estimar a coesão desenvolvida para todas as amostras nesta condição.

\section{RESULTADOS E DISCUSSÕES}

Os resultados da microscopia eletrônica (MEV) são apresentados na Figura 2, a partir das quais pode-se observar uma semelhança no formato das particulas de microcelulose 101 e 102 , porém de formato bastante irregular, as quais se assemelham a fibras alongadas. Já as amostras de lactoses apresentaram morfologias distintas, possuindo a lactose spray-dried um formato mais esférico, enquanto a monohidratada apresenta um formato irregular e um aspecto mais áspero (rugoso), gerando maior área de superficial.

Figura 2: Micrografias: Microceluloses 101, 102, Lactoses Spray-Dried e Monohidratada.
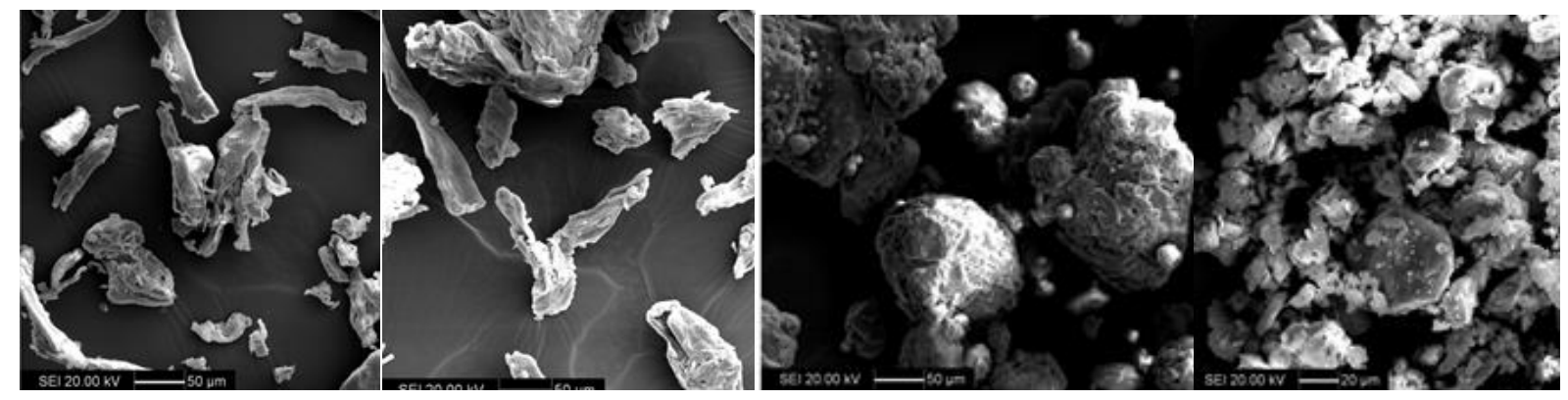

Os resultados da análise granulométrica e das densidades reais, aparentes e compactadas tanto dos excipientes puros estão apresentadas na Tabela 2.

Tabela 2: Tamanho, densidades e porosidades aparentes e compactadas após 2500 taps.

\begin{tabular}{|c|c|c|c|c|c|c|c|c|}
\hline Amostra & $\begin{array}{c}\mathbf{d}_{[4,3]} \mathbf{v o l} \text {. } \\
\mu \mathrm{m}\end{array}$ & $\begin{array}{c}\boldsymbol{\rho}_{\text {real }} \\
\mathrm{g} / \mathrm{cm}^{3}\end{array}$ & $\begin{array}{c}\boldsymbol{\rho}_{\text {apa }} \\
\mathrm{g} / \mathrm{cm}^{3}\end{array}$ & $\boldsymbol{\varepsilon}_{\text {apa }}$ & $\begin{array}{c}\text { Classif. } \\
\text { Haunser }\end{array}$ & $\begin{array}{c}\boldsymbol{\rho}_{\text {comp }} \\
\mathrm{g} / \mathrm{cm}^{3}\end{array}$ & $\boldsymbol{\varepsilon}_{\text {comp }}$ & $\begin{array}{c}\text { Classif. } \\
\text { Haunser }\end{array}$ \\
\hline MCC 101 & 81,21 & 1,573 & 0,503 & 0,768 & Razoável & 0,365 & 0,680 & Pobre \\
\hline MCC 102 & 139,30 & 1,575 & 0,499 & 0,771 & Razoável & 0,360 & 0,683 & Pobre \\
\hline Lact. Mono & 59,40 & 1,545 & 0,719 & 0,664 & Razoável & 0,519 & 0,534 & Pobre \\
\hline Lact. SD & 125,60 & 1,531 & 0,794 & 0,572 & Boa & 0,655 & 0,481 & Razoável \\
\hline
\end{tabular}




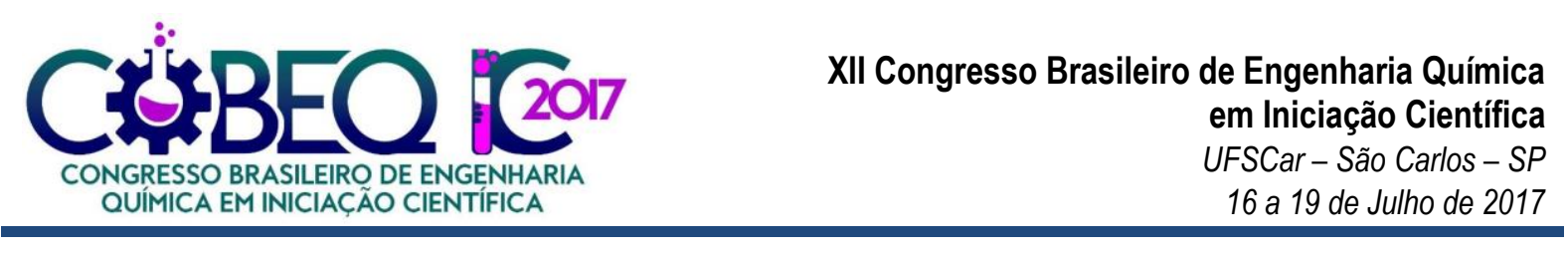

Com os dados das densidades foi possível estimar as porosidades para cada condição das amostras: a denominada porosidade aparente (bulk), na condição de 0 batidas, e a denominada porosidade densa (compactada) na condição de máxima compactação (2500 batidas); e suas respectivas classificações quanto ao índice de Hausner.

Observa-se uma densidade real muito próxima para todos os excipientes, apesar de suas naturezas distintas. Já a densidade aparente, esta aumenta conforme as partículas se aproximam do comportamento esférico, com um comportamento inverso observador para a porosidade.

Em relação a classificação de Hausner, uma piora de todos os materiais foi verificada quando estes são submetidos à compactação. Os resultados podem ser justificados pela hipótese de que uma piora na fluidez é consequência do aumento do número e/ou área de contato entre as partículas, comprovado pela redução no volume ocupado pela amostra e sua porosidade.

Observa-se ainda que esta redução de volume e porosidade da amostra se dá em menor grau para a amostra de Lactose Spray Dried, a qual apresentou melhores índices de fluidez segundo o Índice de Hausner, uma vez que se trata de partículas esféricas.

Os resultados do teste de estabilidade (BFE) e fluxo variável (VRF) para as amostras puras e misturas dos excipientes farmacêuticos estão graficamente ilustrados na Figura 3, sendo que as curvas traçadas representam os valores médios dos ensaios realizados em duplicata.

Figura 3: Resultados dos testes de estabilidade, energia básica de fluxo e de fluxo variável

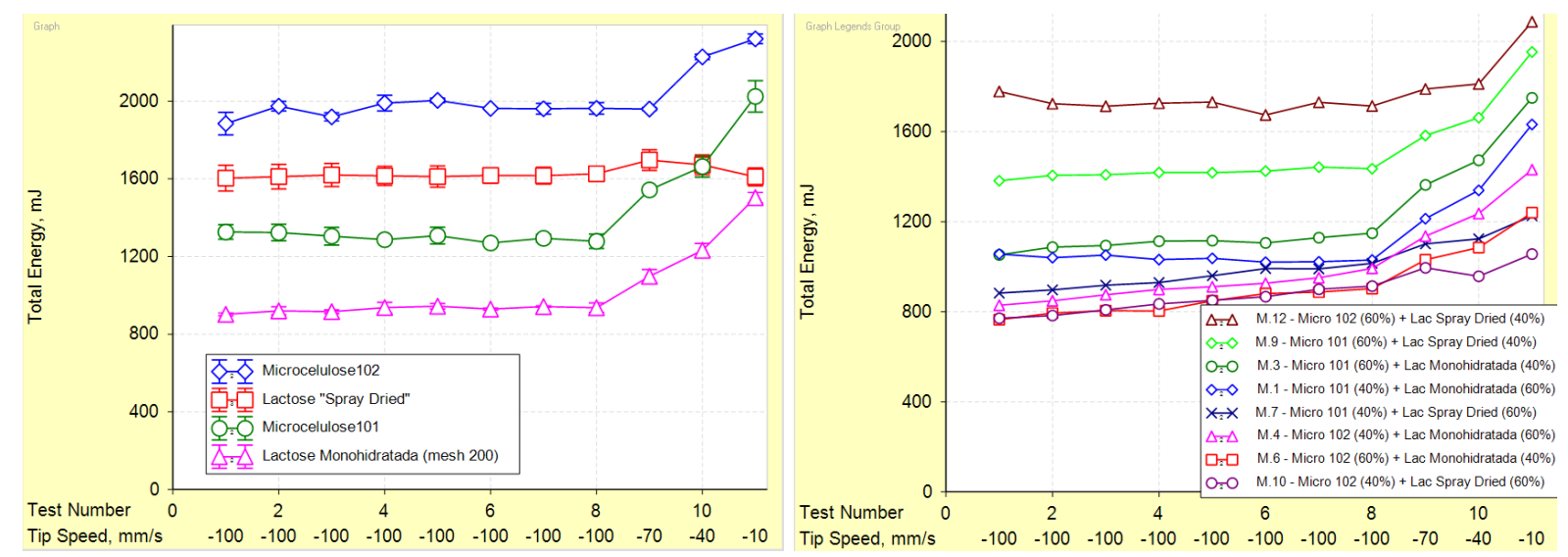

Observa-se que as quatro amostras de excipientes puros são estáveis, ou seja, não há quebra, desaglomeração, segregação ou qualquer outro fenômeno secundário, indicado pelos perfis lineares dos 7 primeiros pontos experimentais de cada pó. Já em relação aos valores da energia básica de fluxo (BFE) destes perfis, observa-se que a microcelulose 102 apresentou a maior energia de fluxo, e a menor foi dispendida pela lactose monohidratada. Esta menor energi se deve a possível formação de aglomerados, com espações vazios no seu interior, característicos de materiais de granulometria reduzida que desenvolvem uma maior coesão.

Em relação ao teste de fluxo variável (4 últimos pontos), a lactose monohidratada e a microcelulose 101 apresentaram os maiores aumentos no valor de BFE com a redução da velocidade de rotação da lâmina (fluxo variável - VRF). Desta forma, estes materiais são mais 


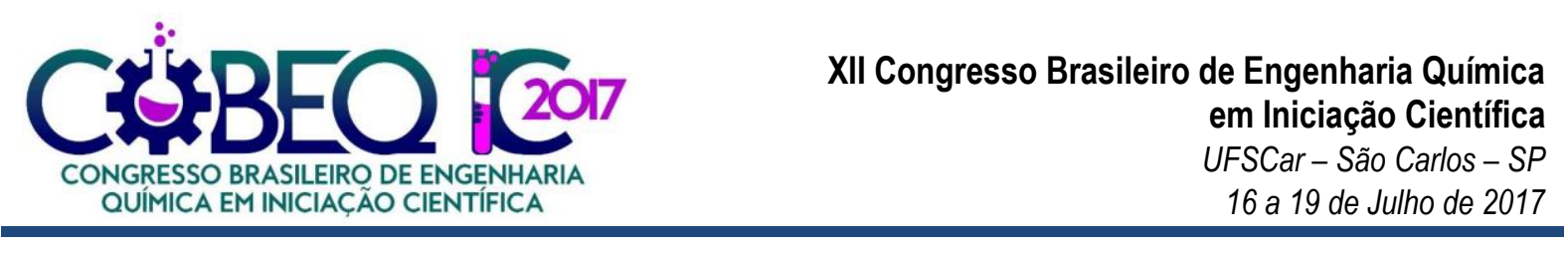

susceptíveis à problemas no processo quando a velocidade de mistura dos equipamentos for reduzida. Já a amostra de lactose spray-dried apresentou uma energia (indicativo de fluidez) constante, independente da velocidade da lâmina, e apresentaram uma fluidez mais uniforme.

Analisando-se o comportamento das misturas, constata-se que as misturas dos excipientes apresentaram valores de BFE inferiores aos observados para os seus componentes puros, com exceção para as misturas de ambas microceluloses com lactose spray-dried no teor de $40 \%$. Aparentemente, este teor não é suficiente para modificar o comportamento reológico das microceluloses. Entretanto, quando o teor de lactose é de $60 \%$, o comportamento sofre grande alteração e a mistura passa a apresentar os menores valores de BFE, indicativo de materiais coesivos, devido a formação de aglomerados (Freeman, 2008).

Em relação a lactose monohidratada, nenhum efeito significativo foi observado quando seu teor passa de $40 \%$ para $60 \%$ nas misturas com ambas microceluloses. Estas continuam a apresentar baixos valores de BFE, indicativo de materiais coesivos.

Os resultados dos testes de aeração dos excipientes puros e de cisalhamento à $3 \mathrm{kPa}$ das misturas, para a determinação da coesão, estão representados graficamente na Figura 4.

Figura 4: Resultados dos testes de aeração e cisalhamento à 3kPa das amostras.
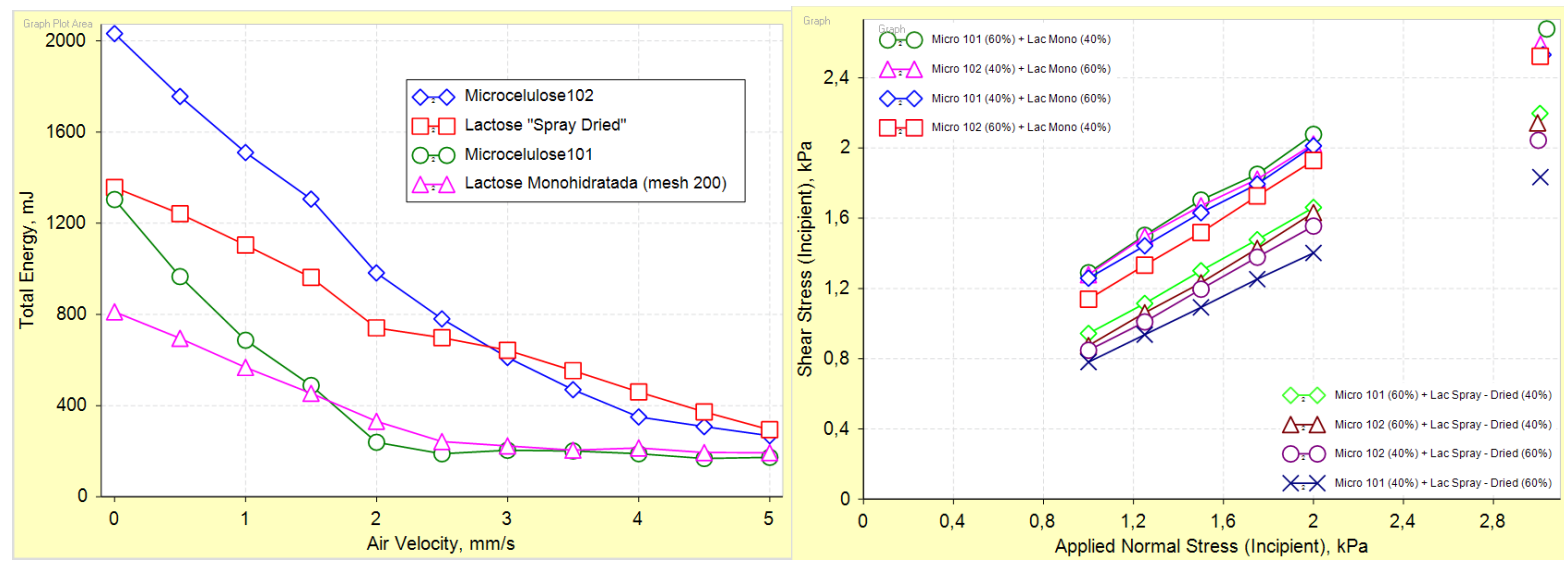

No que se refere ao teste de aeração, apesar da lactose monohidratada apresentar a menor energia BFE no início do teste, esta atinge um patamar constante, diferente de zero em baixas velocidades do ar, assim como a microcelulose 101. Segundo Freeman (2008), esta característica é comum de materiais coesivos e pronunciáveis em materiais de granulometria inferior a $100 \mu \mathrm{m}$, coerentes mais uma vez com os resultados obtidos

Em relação ao teste de cisalhamento, é possível identificar duas regiões distintas, sendo uma superior proveniente das misturas de lactose monohidratada, e uma inferior, caracterizada pelas misturas de lactose spray-dried. Isto indica que a presença de lactose spray-dried (esférica) melhora a fluidez das microceluloses, reduzindo sua coesão, para processos submetidos à tensões de consolidação. 


\section{CONCLUSÃO}

Através dos experimentos realizados foi possível caracterizar as amostras puras e as provenientes das misturas de alguns excipientes farmacêuticos. Em linha gerais, a misturas dos insumos resultou num comportamento distinto e não intermediário às características dos seus constituintes puros.

O aumento das forças coesivas desenvolvida pelo meio granular provavelmente se deve ao aumento do número de contato gerado entre as partículas de diferentes propriedades, sendo a lactose spray-dried a que apresentou a menor coesão, pois são esféricas, de tamanho superior a $100 \mu \mathrm{m}$ e dificilmente o seu número de coordenação sofre alteração.

Entretanto, conforme demonstrado, não é qualquer teor deste material de boa fluidez que pode ser adicionada aos outros insumos para melhorar a fluidez da nova mistura; um estudo se faz necessário para se obter a melhor composição para cada condição de processamento, que neste caso foi $60 \%$ para o estado não consolidado, e de $40 \%$ para o estado consolidado à $3 \mathrm{kPa}$ já o apresenta melhorias significativas.

\section{BIBLIOGRAFIA}

DIETMAR SCHULZE - Flow Properties of Powders and Bulk Solids and Silo Design for Flow. Disponível em <www.dietmar-schulze.de/powtve.pdf>

FERREIRA, V.L.O., Avaliação de funcionalidade de excipientes lubrificantes em comprimidos em compressora instrumentada. Dissertação. FIOCRUZ, Rio de Janeiro, 2013.

FREEMAN TECHNOLOGY, FT-4 Powder Rheometer User's Manual, 2008.

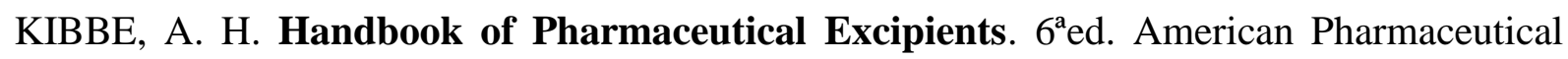
Association, 2009.

PRISTA, L. V. N.; ALVES, A. C.; MORGADO. R.; LOBO, J. S. Tecnologia Farmacêutica. 6 ed. v. 1. Lisboa: Fundação Calouste Gulbenkian, 2003.

SCHULZE, D. Flow Properties of Powders and Bulk Solids. Disponível em: <http://www.dietmar-schulze.de/grdle1.pdf> Acesso em junho 2016.

U.S. Pharmacopeia. Disponível em: <www.pharmacopeia.cn/v29240/usp29nf24s0_c1174.html> Acesso em: Dezembro 2016.

\section{AGRADECIMENTOS}

À empresa Farmanguinhos/RJ pela doação dos insumos farmacêuticos utilizados neste trabalho. 